\title{
Tratamiento antihipertensivo en pacientes de bajo riesgo cardiovascular con hipertensión leve
}

\author{
Antihypertensive treatment in low-risk patients with mild hypertension
}

\section{Objetivos}

Evaluar si el inicio de tratamiento antihipertensivo en pacientes de bajo riesgo cardiovascular (CV) con hipertensión arterial (HTA) leve se asocia con un menor riesgo de muerte y enfermedad cardiovascular.

\section{Diseño}

Cohorte retrospectiva que incluyó 38.286 pacientes (19.143 en cada rama: exposición y control) de 18 a 74 años de edad, con hipertensión leve (140/90 a 159/99 mm Hg) sin historia de enfermedad CV o de sus factores de riesgo y vírgenes de tratamiento farmacológico.

\section{Exposición}

Prescripción de algún fármaco antihipertensivo durante los primeros 12 meses luego del diagnóstico de HTA.

\section{Medición de resultados principales}

La información sobre el tratamiento prescripto (intención de tratar) y sobre otros predictores fue extraída del registro de historias clínicas de atención primaria del Reino Unido. La información de los eventos fue extraída de los certificados de defunción de la Oficina para Estadísticas y de los ingresos hospitalarios (para los eventos CV no fatales) de las Estadísticas de Episodios Hospitalarios.
Sheppard J y col. JAMA Internal Medicine. 2018.

Se llevó a cabo una pseudo-aleatorización de los pacientes para crear dos grupos balanceados, de manera que cada paciente del grupo control estuviera apareado con otro paciente en el grupo exposición con similar probabilidad de que se le haya prescripto tratamiento antihipertensivo. Esta "probabilidad" (en inglés, propensity score) fue calculada en base a variables que pudieran haber inclinado la balanza hacia la prescripción de fármacos antihipertensivos: edad, sexo, etnia, nivel socioeconómico, tabaquismo, consumo de alcohol, índice de masa corporal, valores previos de tensión arterial, artritis reumatoidea, hipercolesterolemia, prescripción de estatinas y antiagregantes, año calendario de inclusión en el estudio y consultorio al que asistía para los controles de salud. El resultado primario fue la mortalidad por todas las causas. Los resultados secundarios fueron muerte u hospitalización por un evento CV mayor (infarto de miocardio, angina inestable, accidente cerebrovascular, insuficiencia cardíaca) y hospitalización por sospecha de efectos adversos de la medicación (hipotensión, síncope, bradicardia, anomalías electrolíticas, caídas o lesión renal aguda).

\section{Resultados principales}

La media del seguimiento fue de 5,8 años. La tabla 1 describe la asociación observada entre el tratamiento antihipertensivo prescripto y los diferentes resultados.

Tabla 1. Asociación observada entre el tratamiento antihipertensivo prescripto y los desenlaces clínicos.

\begin{tabular}{|c|c|c|c|c|}
\hline \multicolumn{2}{|c|}{ Desenlaces } & HR (IC 95\%) & $\mathbf{p}$ & $\begin{array}{l}\text { NND a } 10 \text { años } \\
\text { (IC 95\%) }\end{array}$ \\
\hline \multirow{2}{*}{\multicolumn{2}{|c|}{$\begin{array}{l}\text { Mortalidad } \\
\text { Evento cardiovascular mayor }\end{array}$}} & $1,02(0,88$ a 1,17$)$ & 0,81 & \multirow[t]{2}{*}{ NA } \\
\hline & & $1,09(0,95$ a 1,25$)$ & 0,23 & \\
\hline \multirow{4}{*}{$\begin{array}{l}\text { Sospecha de eventos } \\
\text { adversos atribuibles } \\
\text { al tratamiento }\end{array}$} & Alteraciones electrolíticas & $1,72(1,12$ a 2,65$)$ & 0,01 & 111 (49 a 687) \\
\hline & Hipotensión & $1,69(1,30$ a 2,20$)$ & $<0,001$ & 41 (24 a 93) \\
\hline & Síncope & $1,28(1,10$ a 1,50$)$ & 0,002 & 35 (20 a 100) \\
\hline & Falla renal aguda & $1,37(1$ a 1,88$)$ & 0,048 & 91 (39 a 14552) \\
\hline
\end{tabular}

IC: intervalo de confianza; HR: hazard ratio; NA: no aplica. NND: número de pacientes que es necesario que tengan prescripto el tratamiento para que ocurra un evento adverso adicional.

a No se observó una asociación estadísticamente significativa entre el tratamiento prescripto y la incidencia de caídas ni de bradicardia.

Conclusiones

El tratamiento antihipertensivo en pacientes de bajo riesgo CV e HTA leve no se asoció con una disminución de la mortalidad ni de los eventos CV mayores, pero sí a un mayor riesgo de eventos adversos.

Fuente de financiamiento: National Institute for Health Research.

\section{Comentario}

Tanto las nuevas guías norteamericanas de $2017^{1}$ como las europeas de $2018^{2}$ proponen iniciar tratamiento farmacológico en pacientes con una tensión arterial de $140 / 90 \mathrm{~mm} \mathrm{Hg}$ o mayor, independientemente de su riesgo $\mathrm{CV}$. Sin embargo, las de la Organización Mundial de la Salud ${ }^{3}$, la Sociedad Internacional de Hipertensión y las guías latinoamericanas de hipertensión ${ }^{4}$, plantean recomendar que el paciente adopte hábitos de vida saludables y mantener una conducta expectante en cuanto al tratamiento farmacológico en esta población. Dada la controversia planteada, este estudio observacional intenta brindar información adicional para dirimirla. Sus resultados muestran no sólo que el tratamiento farmacológico no se traduciría en un beneficio en términos de reducción de la mortalidad y/o de eventos CV mayores, sino que además expondría a esta población a un mayor riesgo de padecer eventos adversos con la terapia a largo plazo; detalle no menor, ya que -de cumplir con las indicaciones de las guías norteamericanas y europeas- serían medicadas millones de personas e inevitablemente muchas de ellas resultarían dañadas sin un beneficio claro.

\section{Conclusión de la comentadora}

Si bien la calidad de la evidencia de este estudio no es comparable con la de los ensayos clínicos, su sofisticado y prolijo diseño permite obtener resultados valiosos.

Leticia Gavoto [ Servicio de Medicina Familiar y Comunitaria, Hospital Italiano de Buenos Aires. leticia.gavoto@ hospitalitaliano.org.ar ]

Gavoto L. Tratamiento antihipertensivo en pacientes de bajo riesgo cardiovascular con hipertensión leve. Evid Actual Práct Ambul. 2018;21(4):113. Comentado de: Sheppard J y col. Benefits and Harms of Antihypertensive Treatment in Low-Risk Patients With Mild Hypertension. JAMA Internal Medicine. 2018;178(12):1626-1634. PMID:30383082

\section{Referencias}

1. Whelton PK y col. 2017 ACC/AHA/AAPA/ABC/ACPM/AGS/APhA/ASH/ASPC/NMA/PCNA Guideline for the Prevention, Detection, Evaluation, and Management of High Blood Pressure in Adults: A Report of the American College of Cardiology/American Heart Association Task Force on Clinical Practice Guidelines. J Am Coll Cardiol. 2018;71(19):e127-e248.

2. Williams B y col. 2018 ESC/ESH Guidelines for the management of arterial hypertension. Eur Heart J. 2018;39(33):3021-3104.

3. Whitworth JA, World Health Organization, International Society of Hypertension Writing Group. 2003 World Health Organization (WHO)/International Society of Hypertension (ISH) statement on management of hypertension. J Hypertens. 2003;21(11):1983-92.

4. Sanchez RA y col. Latin American guidelines on hypertension. Latin American Expert Group. J Hypertens. 2009;27(5):905-22. 